\title{
A SHORT PROOF OF WEYL'S LAW FOR FRACTIONAL DIFFERENTIAL OPERATORS
}

\author{
LEANDER GEISINGER
}

\begin{abstract}
We study spectral asymptotics for a large class of differential operators on an open subset of $\mathbb{R}^{d}$ with finite volume. This class includes the Dirichlet Laplacian, the fractional Laplacian, and also fractional differential operators with non-homogeneous symbols. Based on a sharp estimate for the sum of the eigenvalues we establish the first term of the semiclassical asymptotics. This generalizes Weyl's law for the Laplace operator.
\end{abstract}

\section{INTRODUCTION AND MAIN RESUlT}

In this note we study the asymptotic distribution of eigenvalues of a differential operator $\mathcal{L}_{T}$ with symbol $T: \mathbb{R}^{d} \rightarrow \mathbb{R}$ on an open set $\Omega \subset \mathbb{R}^{d}$ of finite volume. We define the operator $\mathcal{L}_{T}$ in terms of the quadratic form

$$
Q_{T}[u]=\int_{\mathbb{R}^{d}} T(p)|\hat{u}(p)|^{2} d p
$$

restricted to the form domain

$$
\mathcal{H}_{T}(\Omega)=\left\{u \in L^{2}\left(\mathbb{R}^{d}\right): \int_{\mathbb{R}^{d}}(1+T(p))|\hat{u}(p)|^{2} d p<\infty \text { and } u \equiv 0 \text { on } \mathbb{R}^{d} \backslash \Omega\right\},
$$

where $\hat{u}(p)=(2 \pi)^{-d / 2} \int e^{-i p \cdot x} u(x) d x$ is the Fourier transform of $u$. Then $\mathcal{L}_{T}$ is defined to be the self-adjoint operator satisfying $Q_{T}[u]=\left\langle u, \mathcal{L}_{T} u\right\rangle$, where $\langle\cdot, \cdot\rangle$ denotes the scalar product in $L^{2}\left(\mathbb{R}^{d}\right)$.

For example, for $T(p)=|p|^{2}$ we obtain the Dirichlet Laplace operator, with form domain given by the Sobolev space $H_{0}^{1}(\Omega)$. More generally, for $T(p)=|p|^{2 s}, 0<s \leq 1, \mathcal{L}_{T}$ is the fractional Laplacian $(-\Delta)^{s}$, see for example [4] for details concerning the definition.

For the Dirichlet Laplacian spectral asymptotics are well-known: For $\Omega \subset \mathbb{R}^{d}$ of finite volume the spectrum is discrete and consists of eigenvalues $0<\lambda_{1} \leq \lambda_{2} \leq \ldots$ with finite multiplicities. In 1912, Weyl proved the famous asymptotic law [18]

$$
\lambda_{n}=\frac{4 \pi \Gamma(1+d / 2)^{2 / d}}{|\Omega|^{2 / d}} n^{2 / d}(1+o(1)), \quad n \rightarrow \infty,
$$

initially for a bounded domain in two dimensions. Here $|\Omega|$ denotes the volume of $\Omega$ and $\Gamma$ is the gamma function. This result was later improved and generalized in various ways, see for example [2, 9, 10, 17] for a summary of results and applications concerning the Laplace operator and further references.

The purpose of this note is to give a short proof of the analogue of this result for the operator $\mathcal{L}_{T}$ under minimal assumptions on the set $\Omega$ (we only require that the volume is finite) and under weak assumptions on the symbol $T$. The method is elementary, in particular, we do not use bracketing as in [16, Thm. XIII.78] and we need only a simple

Date: September 10, 2013.

(C) 2013 by the author. This paper may be reproduced, in its entirety, for non-commercial purposes. 
version of a Tauberian theorem, see Lemma 3 below. Along the way, in Theorem 2 , we prove a sharp bound on the sum of the eigenvalues of $\mathcal{L}_{T}$, an analogue of the Berezin-Li-Yau inequality for the Dirichlet Laplacian [1,13].

We assume that $T$ satisfies the following conditions:

I There is a function $T_{0}: \mathbb{R}^{d} \rightarrow \mathbb{R}$ with the following three properties. $T_{0}$ is homogeneous of degree $\alpha>0$ : $T_{0}(\nu p)=\nu^{\alpha} T_{0}(p)$ for $p \in \mathbb{R}^{d}$ and $\nu>0$. The set of $p \in \mathbb{R}^{d}$ with $T_{0}(p)<1$ has finite Lebesgue-measure:

$$
V_{T}:=\left|\left\{p \in \mathbb{R}^{d}: T_{0}(p)<1\right\}\right|<\infty .
$$

The function $T_{0}$ approximates $T$ in the sense that for all $p \in \mathbb{R}^{d}$

$$
\lim _{\nu \rightarrow \infty} \nu^{-\alpha} T(\nu p)=T_{0}(p)
$$

Moreover, if this convergence is not uniform in $p \in \mathbb{R}^{d}$ we also require that there is a function $\tilde{T}: \mathbb{R}^{d} \rightarrow \mathbb{R}$ which is locally integrable, larger than 1 at infinity (i.e. there is $R>0$ such that $\tilde{T}(p) \geq 1$ for $|p| \geq R$ ), and such that there is $\nu_{0}>0$ with $\tilde{T}(p) \leq \nu^{-\alpha} T(\nu p)$ for all $p \in \mathbb{R}^{d}$ and $\nu \geq \nu_{0}$.

II There are constants $C_{0}>0$ and $N \in \mathbb{N}$ such that for all $\eta \in \mathbb{R}^{d}$

$$
\sup _{p \in \mathbb{R}^{d}}\left(\frac{1}{2}(T(p+\eta)+T(p-\eta))-T(p)\right) \leq C_{0}(1+|\eta|)^{N}
$$

Under these assumptions, since the volume of $\Omega$ is finite, the spectrum of $\mathcal{L}_{T}$ is discrete and consists of positive eigenvalues with finite multiplicities. We denote these eigenvalues by $\left(\lambda_{k}\right)_{k \in \mathbb{N}}$. To study the asymptotic distribution we introduce the counting function $N(\Lambda)$ that counts the number of eigenvalues below $\Lambda>0$ :

$$
N(\Lambda)=\sum_{k \in \mathbb{N}}\left(\Lambda-\lambda_{k}\right)_{+}^{0}=\operatorname{Tr}\left(\mathcal{L}_{T}-\Lambda\right)_{-}^{0} .
$$

Here $x_{ \pm}=(|x| \pm x) / 2$ denotes the positive and negative part of $x \in \mathbb{R}$ and we use the same convention for self-adjoint operators.

Theorem 1. Let $\Omega \subset \mathbb{R}^{d}$ be an open set of finite volume and assume that the symbol $T$ satisfies assumptions $\square$ and $\llbracket$. Then, as $\Lambda \rightarrow \infty$, the asymptotic formula

$$
\lim _{\Lambda \rightarrow \infty} \Lambda^{-d / \alpha} N(\Lambda)=(2 \pi)^{-d}|\Omega| V_{T}
$$

holds.

The assumptions [ and [I are satisfied by the Dirichlet Laplacian and more generally by the fractional Laplacian $(-\Delta)^{s}$ with symbol $T(p)=|p|^{2 s}, 0<s \leq 1$. In this case the symbol itself is homogeneous and we have $T=T_{0}$ in assumption 【. For the fractional Laplacian Theorem 1 was proved by Blumenthal and Getoor under additional assumptions on the boundary of $\Omega[3]$.

We emphasize that the theorem also applies to non-homogeneous symbols, for example to the operator with symbol $T(p)=|p|^{\alpha} \pm|p|^{\beta}, 0<\beta<\alpha \leq 2$. In this case we have $T_{0}(p)=|p|^{\alpha}$ and we can choose $\tilde{T}(p)=T_{0}(p)$ for $T(p)=|p|^{\alpha}+|p|^{\beta}$ and $\tilde{T}(p)=T(p)$ for $T(p)=|p|^{\alpha}-|p|^{\beta}$. 
To illustrate the result let us consider the fractional differential operator

$$
\mathcal{L}_{2 s}=\sum_{i=1}^{d}\left(-\partial_{i}^{2}\right)^{s}, \quad 0<s \leq 1,
$$

that was recently studied in [8]. This operator corresponds to $\mathcal{L}_{T}$ with symbol $T(p)=$ $\sum_{i=1}^{d}\left|p_{i}\right|^{2 s}=\|p\|_{2 s}^{2 s}$ and is the generator of a Levy-process killed upon exiting $\Omega$. In [8] it is proved that (1) holds for $\mathcal{L}_{2 s}$ but only if $\Omega$ is a hypercube. Theorem 1 shows that this can be generalized to arbitrary open sets $\Omega$ of finite volume.

Indeed, we note that (see [8])

$$
V_{\|p\|_{2 s}^{2 s}}=\int_{\mathbb{R}^{d}}\left(\|p\|_{2 s}^{2 s}-1\right)_{-}^{0} d p=\frac{(2 \Gamma(1+1 / 2 s))^{d}}{\Gamma(1+d / 2 s)} .
$$

Moreover, observe that for $0<s \leq 1$ and all $r \in[0,1]$, by concavity,

$$
r^{2 s}+1-\frac{1}{2}\left(\left(1+r^{2}+2 r\right)^{s}+\left(1+r^{2}-2 r\right)^{s}\right) \geq r^{2 s}+1-\left(r^{2}+1\right)^{s} \geq 0 .
$$

It follows that, for all $t \in \mathbb{R},\left(|1+t|^{2 s}+|1-t|^{2 s}\right) / 2-|t|^{2 s} \leq 1$. Thus, for all $p_{i} \in \mathbb{R}$ and $\eta_{i} \in \mathbb{R}$

$$
\sum_{i=1}^{d}\left(\frac{1}{2}\left(\left|p_{i}+\eta_{i}\right|^{2 s}+\left|p_{i}-\eta_{i}\right|^{2 s}\right)-\left|p_{i}\right|^{2 s}\right) \leq \sum_{i=1}^{d}\left|\eta_{i}\right|^{2 s} .
$$

We see that the operator $\mathcal{L}_{2 s}$ satisfies conditions $\square$ and $\amalg$ and from Theorem 1 we obtain

$$
\lim _{\Lambda \rightarrow \infty} N(\Lambda) \Lambda^{-d / 2 s}=\frac{(2 \Gamma(1+1 / 2 s))^{d}}{\Gamma(1+d / 2 s)} \frac{|\Omega|}{(2 \pi)^{d}} .
$$

For $s=1$ we recover the result of Weyl [18] about the Laplacian.

\section{Auxiliary Results}

Our method of proof is rather general and applicable in other situations as well. We follow the strategy of [7], where an operator with magnetic field is considered. This approach is based on an application of coherent states. Moreover, we rely on the following sharp spectral estimate which is the analogue of the Berezin-Li-Yau inequality for the Dirichlet Laplacian [1,13,14, see also [12].

Theorem 2. For $\Omega \subset \mathbb{R}^{d}$ of finite volume and all $\Lambda>0$

$$
\operatorname{Tr}\left(\mathcal{L}_{T}-\Lambda\right)_{-} \leq \frac{|\Omega|}{(2 \pi)^{d}} \int_{\mathbb{R}^{d}}(T(p)-\Lambda)_{-} d p
$$

Proof. We follow the proof given in [5,6] for the Laplacian and the fractional Laplacian. Let $\chi_{\Omega}$ denote the characteristic function of $\Omega$. Then due to the definition of $\mathcal{L}_{T}$ (note that it is defined on functions that are zero on the complement of $\Omega$ )

$$
\operatorname{Tr}\left(\mathcal{L}_{T}-\Lambda\right)_{-}=\operatorname{Tr}\left(\chi_{\Omega}\left(\mathcal{L}_{T}-\Lambda\right) \chi_{\Omega}\right)_{-} \leq \operatorname{Tr}\left(\chi_{\Omega}\left(\mathcal{L}_{T}-\Lambda\right)_{-} \chi_{\Omega}\right),
$$

where the second relation follows from the variational principle. Now we can write out the kernel of the operator on the right and calculate its trace. We obtain

$$
\begin{aligned}
\operatorname{Tr}\left(\mathcal{L}_{T}-\Lambda\right)_{-} & \leq \frac{1}{(2 \pi)^{d}} \int_{\mathbb{R}^{d}}\left[\chi_{\Omega}(x) \int_{\mathbb{R}^{d}} e^{i p \cdot(x-y)}(T(p)-\Lambda)_{-} d p \chi_{\Omega}(y)\right]_{x=y} d x \\
& =\frac{|\Omega|}{(2 \pi)^{d}} \int_{\mathbb{R}^{d}}(T(p)-\Lambda)_{-} d p
\end{aligned}
$$


and the proof is complete.

If the symbol $T(p)$ is homogeneous of degree $\alpha$, so that $T(p)=T_{0}(p)$ in assumption 【, then we change variables $p=\mu^{1 / \alpha} \xi, \mu>0$, and by Fubini's theorem we get

$$
\begin{aligned}
\int_{\mathbb{R}^{d}}(T(p)-\Lambda)_{-} d p & =\int_{\mathbb{R}^{d}} \int_{0}^{\Lambda}(T(p)-\mu)_{-}^{0} d \mu d p \\
& =\int_{0}^{\Lambda} \mu^{d / \alpha} \int_{\mathbb{R}^{d}}\left(\mu^{-1} T\left(\mu^{1 / \alpha} \xi\right)-1\right)_{-}^{0} d \xi d \mu \\
& =\frac{\alpha}{d+\alpha} V_{T} \Lambda^{1+d / \alpha} .
\end{aligned}
$$

Hence for homogeneous symbols we obtain from Theorem 2

$$
\operatorname{Tr}\left(\mathcal{L}_{T}-\Lambda\right)_{-} \leq \frac{\alpha}{d+\alpha} \frac{|\Omega|}{(2 \pi)^{d}} V_{T} \Lambda^{1+d / \alpha}
$$

for all $\Lambda>0$. We note that $\operatorname{Tr}\left(\mathcal{L}_{T}-\Lambda\right)_{-}=\sum_{k}\left(\Lambda-\lambda_{k}\right)_{+}=\int_{0}^{\Lambda} N(\mu) d \mu$. Inserting the asymptotic result from Theorem 1 we see that (3) yields a sharp bound.

In fact, (3) is equivalent to a sharp lower bound on the sum of the eigenvalues. Note that, for all $M \in \mathbb{N}$,

$$
\sum_{k=1}^{M} \lambda_{k}=\sup _{\Lambda>0}\left(M \Lambda-\sum_{k \in \mathbb{N}}\left(\Lambda-\lambda_{k}\right)_{+}\right)
$$

so that, by (3),

$$
\sum_{k=1}^{M} \lambda_{k} \geq \frac{d}{d+\alpha} \frac{(2 \pi)^{\alpha}}{\left(|\Omega| V_{T}\right)^{\alpha / d}} M^{1+\alpha / d} .
$$

The bounds (3) and (4) are generalizations of the Berezin-Li-Yau inequality for the Dirichlet Laplacian. For the operator $\mathcal{L}_{2 s}$ we obtain the bound

$$
\sum_{k=1}^{M} \lambda_{k} \geq \frac{d}{d+2 s} \frac{(2 \pi)^{2 s} \Gamma(1+d / 2 s)^{2 s / d}}{(2 \Gamma(1+1 / 2 s))^{2 s}}|\Omega|^{2 s / d} M^{1+2 s / d}
$$

that was derived in [8] using the arguments from [13].

We also need the following well-known lemma, see for example [11, Lemma 17.1], which is a weak, elementary version of a Tauberian theorem. For completeness we include the short proof.

Lemma 3. Let $\left(\xi_{k}\right)_{k \in \mathbb{N}}$ be a non-decreasing sequence of positive numbers that tends to infinity. Assume that there are finite constants $A>0$ and $a>0$ such that

$$
\lim _{\Lambda \rightarrow \infty} \Lambda^{-a-1} \sum_{k \in \mathbb{N}}\left(\Lambda-\xi_{k}\right)_{+}=A .
$$

Then

$$
\lim _{\Lambda \rightarrow \infty} \Lambda^{-a} \sum_{k \in \mathbb{N}}\left(\Lambda-\xi_{k}\right)_{+}^{0}=(a+1) A
$$

Proof. Let us introduce the notation $S(\Lambda)=\sum_{k}\left(\Lambda-\xi_{k}\right)_{+}$and $N(\Lambda)=\sum_{k}\left(\Lambda-\xi_{k}\right)_{+}^{0}$. For any $h>0$ and $\Lambda>0, k \in \mathbb{N}$ we have $\left(\Lambda+h-\xi_{k}\right)_{+}-\left(\Lambda-\xi_{k}\right)_{+} \geq h\left(\Lambda-\xi_{k}\right)_{+}^{0}$, thus

$$
S(\Lambda+h)-S(\Lambda) \geq h N(\Lambda) .
$$


By assumption, for fixed $0<\epsilon \leq 1$, we find $\Lambda$ large enough such that $\left|\Lambda^{-a-1} S(\Lambda)-A\right| \leq \epsilon$. Hence, for $0<h \leq \Lambda$ relation (5) implies

$$
\begin{aligned}
N(\Lambda) & \leq \frac{1}{h}\left(A\left((\Lambda+h)^{a+1}-\Lambda^{a+1}\right)+\epsilon\left((\Lambda+h)^{a+1}+\Lambda^{a+1}\right)\right) \\
& \leq(a+1) A \Lambda^{a}+C \Lambda^{a-1} h+\frac{\epsilon}{h}\left(2^{a+1}+1\right) \Lambda^{a+1}
\end{aligned}
$$

with a constant $C>0$ depending only on $A$ and $a$. Now we choose $h=\sqrt{\epsilon} \Lambda$ and obtain

$$
\Lambda^{-a} N(\Lambda) \leq(a+1) A+\sqrt{\epsilon}\left(C+\left(2^{a+1}+1\right)\right) .
$$

Since $0<\epsilon \leq 1$ was arbitrary this completes the proof of the upper bound. The lower bound follows similarly using the fact that $\left(\Lambda-\xi_{k}\right)_{+}-\left(\Lambda-h-\xi_{k}\right)_{+} \leq h\left(\Lambda-\xi_{k}\right)_{+}^{0}$ for $h>0$.

\section{Proof of the MAIN RESUlt}

With Theorem 2 and Lemma 3 at hand we can now give the proof of Theorem 1. By Lemma 3 and the fact that $\operatorname{Tr}\left(\mathcal{L}_{T}-\Lambda\right)_{-}=\sum_{k}\left(\Lambda-\lambda_{k}\right)_{+}$it suffices to prove the asymptotics

$$
\operatorname{Tr}\left(\mathcal{L}_{T}-\Lambda\right)_{-}=\frac{\alpha}{\alpha+d} \frac{|\Omega|}{(2 \pi)^{d}} V_{T} \Lambda^{1+d / \alpha}(1+o(1)), \quad \Lambda \rightarrow \infty
$$

The upper bound follows from Theorem 2. Indeed, writing $p=\Lambda^{1 / \alpha} \xi$ we get

$$
\operatorname{Tr}\left(\mathcal{L}_{T}-\Lambda\right)_{-} \leq \frac{|\Omega|}{(2 \pi)^{d}} \int_{\mathbb{R}^{d}}(T(p)-\Lambda)_{-} d p=\frac{|\Omega|}{(2 \pi)^{d}} \int_{\mathbb{R}^{d}}\left(\Lambda^{-1} T\left(\Lambda^{1 / \alpha} \xi\right)-1\right)_{-} d \xi \Lambda^{1+d / \alpha} .
$$

By assumption \, dominated convergence shows that

$$
\operatorname{Tr}\left(\mathcal{L}_{T}-\Lambda\right)_{-} \leq \frac{|\Omega|}{(2 \pi)^{d}} \int_{\mathbb{R}^{d}}\left(T_{0}(\xi)-1\right)_{-} d \xi \Lambda^{1+d / \alpha}(1+o(1))
$$

as $\Lambda \rightarrow \infty$. Hence, the upper bound in (6) follows from the identity

$$
\int_{\mathbb{R}^{d}}\left(T_{0}(\xi)-1\right)_{-} d \xi=\frac{\alpha}{\alpha+d} V_{T}
$$

which is derived in the same way as equation (2) in Section 2 ,

To prove the lower bound fix $\delta>0$ and put $\Omega_{\delta}=\left\{x \in \Omega: \operatorname{dist}\left(x, \mathbb{R}^{d} \backslash \Omega\right)>\delta\right\}$. By dominated convergence, $\left|\Omega_{\delta}\right| \rightarrow|\Omega|$ as $\delta \rightarrow 0$, hence it suffices to show the lower bound in (6) with $\Omega$ replaced by $\Omega_{\delta}$.

Let $g \in C_{0}^{\infty}\left(\mathbb{R}^{d}\right)$ be a real-valued, $L^{2}$-normalized function with support in $\left\{x \in \mathbb{R}^{d}:|x| \leq\right.$ $\delta / 2\}$. For $p \in \mathbb{R}^{d}$ and $q \in \Omega_{\delta}$ we introduce the coherent states $F_{p, q}(x)=e^{i p \cdot x} g(x-q)$. Then the properties of coherent states (see, e.g, [15, Thm. 12.8]) imply

$$
\operatorname{Tr}\left(\mathcal{L}_{T}-\Lambda\right)_{-} \geq \frac{1}{(2 \pi)^{d}} \iint_{\mathbb{R}^{d} \times \Omega_{\delta}}\left\langle F_{p, q},\left(\mathcal{L}_{T}-\Lambda\right)_{-} F_{p, q}\right\rangle d p d q .
$$

Note that the map $t \mapsto(t-\Lambda)_{-}$is convex and that $\left\|F_{p, q}\right\|_{L^{2}\left(\mathbb{R}^{d}\right)}=1$. Thus we can apply Jensen's inequality to the spectral measure of $\mathcal{L}_{T}$ and obtain

$$
\operatorname{Tr}\left(\mathcal{L}_{T}-\Lambda\right)_{-} \geq \frac{1}{(2 \pi)^{d}} \iint_{\mathbb{R}^{d} \times \Omega_{\delta}}\left(\left\langle F_{p, q}, \mathcal{L}_{T} F_{p, q}\right\rangle-\Lambda\right)_{-} d p d q
$$

We claim that there is a constant $C>0$ (independent of $p, q$, and $\Lambda$ ) such that

$$
\left\langle F_{p, q}, \mathcal{L}_{T} F_{p, q}\right\rangle \leq T(p)+C .
$$


Then, after inserting this estimate into the bound above we can integrate over $q \in \Omega_{\delta}$ and find

$$
\operatorname{Tr}\left(\mathcal{L}_{T}-\Lambda\right)_{-} \geq \frac{\left|\Omega_{\delta}\right|}{(2 \pi)^{d}} \int_{\mathbb{R}^{d}}(T(p)+C-\Lambda)_{-} d p .
$$

In the same way as above the relations

$$
\begin{aligned}
\int_{\mathbb{R}^{d}}(T(p)+C-\Lambda)_{-} d p & =\int_{\mathbb{R}^{d}}\left(\Lambda^{-1} T\left(\Lambda^{1 / \alpha} \xi\right)+\Lambda^{-1} C-1\right)_{-} d \xi \Lambda^{1+d / \alpha} \\
& =\int_{\mathbb{R}^{d}}\left(T_{0}(\xi)-1\right)_{-} d \xi \Lambda^{1+d / \alpha}(1+o(1)) \\
& =\frac{\alpha}{d+\alpha} V_{T} \Lambda^{1+d / \alpha}(1+o(1))
\end{aligned}
$$

follow from dominated convergence and the homogeneity of $T_{0}$ by assumption \. Thus we conclude

$$
\liminf _{\Lambda \rightarrow \infty} \Lambda^{-1-d / \alpha} \operatorname{Tr}\left(\mathcal{L}_{T}-\Lambda\right)_{-} \geq \frac{\alpha}{d+\alpha} \frac{\left|\Omega_{\delta}\right|}{(2 \pi)^{d}} V_{T} .
$$

This is the required lower bound of ([6). Hence, to complete the proof of Theorem 1 it remains to establish (8).

By definition of $\mathcal{L}_{T}$ and $F_{p, q}$ we have

$$
\left\langle F_{p, q}, \mathcal{L}_{T} F_{p, q}\right\rangle=\frac{1}{(2 \pi)^{d}} \iiint e^{i(p-\xi) \cdot(x-y)} g_{q}(x) g_{q}(y) T(\xi) d x d y d \xi
$$

where we write $g_{q}(x)=g(x-q)$ and all integrals are taken over $\mathbb{R}^{d}$. We insert the identity $g_{q}(x) g_{q}(y)=\left(g_{q}(x)^{2}+g_{q}(y)^{2}-\left(g_{q}(x)-g_{q}(y)\right)^{2}\right) / 2$ and use the symmetry of the integral above to obtain

$$
\begin{aligned}
\left\langle F_{p, q}, \mathcal{L}_{T} F_{p, q}\right\rangle & =\iiint e^{i(p-\xi) \cdot(x-y)}\left(g_{q}(x)^{2}-\frac{1}{2}\left(g_{q}(x)-g_{q}(y)\right)^{2}\right) T(\xi) \frac{d x d y d \xi}{(2 \pi)^{d}} \\
& =T(p)-\iiint e^{i(p-\xi) \cdot(x-y)}\left(g_{q}(x)-g_{q}(y)\right)^{2} T(\xi) \frac{d x d y d \xi}{2(2 \pi)^{d}}
\end{aligned}
$$

where in the second step we used the fact that $\int g(x-q)^{2} d x=1$ for all $q \in \mathbb{R}^{d}$.

To estimate the contribution of the second summand we substitute $y=x-z$ and apply Plancherel's Theorem to get

$$
\begin{aligned}
& \iiint e^{i(p-\xi) \cdot z}\left(g_{q}(x)-g_{q}(x-z)\right)^{2} T(\xi) \frac{d x d z d \xi}{2(2 \pi)^{d}} \\
& =\iiint e^{i(p-\xi) \cdot z}\left|\hat{g}_{q}(\eta)\right|^{2}\left|1-e^{-i z \cdot \eta}\right|^{2} T(\xi) \frac{d \eta d z d \xi}{2(2 \pi)^{d}} .
\end{aligned}
$$

We note that $\left|\hat{g}_{q}(\eta)\right|^{2}=|\hat{g}(\eta)|^{2}$ is independent of $q$. Moreover, we write $\left|1-e^{-i z \cdot \eta}\right|^{2}=$ $2-e^{i z \cdot \eta}-e^{-i z \cdot \eta}$ and perform the integration in $z$ and $\xi$. We find

$$
\begin{aligned}
& \iiint e^{i(p-\xi) \cdot(x-y)}\left(g_{q}(x)-g_{q}(y)\right)^{2} T(\xi) \frac{d x d y d \xi}{2(2 \pi)^{d}} \\
& =\int\left(T(p)-\frac{1}{2}(T(p+\eta)+T(p-\eta))\right)|\hat{g}(\eta)|^{2} d \eta .
\end{aligned}
$$

By assumption $\prod$ the right-hand side is bounded below by $-C_{0} \int(1+|\eta|)^{N}|\hat{g}(\eta)|^{2} d \eta \geq-C$. Combining this estimate with (9) yields (8) and completes the proof of Theorem 1, 
Acknowledgments. It is a pleasure to thank Rupert L. Frank and Anna Vershynina for helpful comments. Financial support from DFG grant GE 2369/1-1 and NSF grant PHY1122309 is gratefully acknowledged.

\section{REFERENCES}

[1] F. A. Berezin, Convex functions of operators, Mat. Sb. (N.S.) 88(130) (1972), 268-276.

[2] M. S. Birman and M. Z. Solomjak, Quantitative analysis in Sobolev imbedding theorems and applications to spectral theory, American Mathematical Society Translations, Series 2, vol. 114, American Mathematical Society, Providence, R.I., 1980.

[3] R. M. Blumenthal and R. K. Getoor, The asymptotic distribution of the eigenvalues for a class of Markov operators, Pacific J. Math. 9 (1959), 399-408.

[4] Eleonora Di Nezza, Giampiero Palatucci, and Enrico Valdinoci, Hitchhiker's guide to the fractional Sobolev spaces, Bull. Sci. Math. 136 (2012), no. 5, 521-573.

[5] R. L. Frank and L. Geisinger, Refined semiclassical asymptotics for fractional powers of the Laplace operator, preprint: arXiv:1105:5181.

[6] _ Two-term spectral asymptotics for the Dirichlet Laplacian on a bounded domain, Mathematical results in quantum physics, World Sci. Publ., Hackensack, NJ, 2011, pp. 138-147.

[7] Rupert L. Frank, Remarks on eigenvalue estimates and semigroup domination, Spectral and scattering theory for quantum magnetic systems, Contemp. Math., vol. 500, Amer. Math. Soc., Providence, RI, 2009, pp. 63-86.

[8] Agapitos N. Hatzinikitas, Spectral properties of the Dirichlet operator $\sum_{i=1}^{d}\left(-\partial_{i}^{2}\right)^{s}$ on domains in $d$ dimensional Euclidean space, preprint: arXiv:1301.4806.

[9] L. Hörmander, The analysis of linear partial differential operators, vol. 4, Springer-Verlag, Berlin, 1985.

[10] V. Ja. Ivrii, Microlocal analysis and precise spectral asymptotics, Springer Monographs in Mathematics, Springer-Verlag, Berlin, 1998.

[11] Jacob Korevaar, Tauberian theory, Grundlehren der Mathematischen Wissenschaften [Fundamental Principles of Mathematical Sciences], vol. 329, Springer-Verlag, Berlin, 2004, A century of developments.

[12] A. Laptev, Dirichlet and Neumann eigenvalue problems on domains in Euclidean spaces, J. Funct. Anal. 151 (1997), no. 2, 531-545.

[13] P. Li and S. T. Yau, On the Schrödinger equation and the eigenvalue problem, Comm. Math. Phys. 88 (1983), no. 3, 309-318.

[14] E. H. Lieb, The classical limit of quantum spin systems, Comm. Math. Phys. 31 (1973), 327-340.

[15] Elliott H. Lieb and Michael Loss, Analysis, second ed., Graduate Studies in Mathematics, vol. 14, American Mathematical Society, Providence, RI, 2001.

[16] M. Reed and B. Simon, Methods of Modern Mathematical Physics IV. Analysis of Operators, Academic Press, 1978.

[17] Y. Safarov and D. Vassiliev, The asymptotic distribution of eigenvalues of partial differential operators, Translations of Mathematical Monographs, 155, American Mathematical Society, Providence, RI, 1997.

[18] H. Weyl, Das asymptotische Verteilungsgesetz der Eigenwerte linearer partieller Differentialgleichungen (mit einer Anwendung auf die Theorie der Hohlraumstrahlung), Math. Ann. 71 (1912), no. 4, 441-479.

Leander Geisinger, Department of Physics, Princeton University, Princeton, NJ 08544, USA

E-mail address: leander@princeton.edu 